\title{
Series form solution for a graded composite strip with elastic variation in span direction
}

\author{
Qing Yang ${ }^{1,2, a}{ }^{*}$, Weiping Liu ${ }^{1, b}$, Muhuo $\mathrm{Yu}^{2, \mathrm{c}}$, Dongyan Xiu ${ }^{1}$, Ping Cheng ${ }^{1}$, \\ Lijie Jia ${ }^{1}$, Peng $X u^{1}$, Ran Wei ${ }^{1}$, Jingjing Sun ${ }^{1}$
}

1. Shanghai Aircraft Manufacturing Co., Ltd, COMAC, Shanghai, 200436;

2. College of Materials Science and Engineering, Donghua University, Shanghai, 201620

yangqing3@comac.cc; liuweipin@comac.cc; yumuhuo@dhu.edu.cn

Key words: Series solution, Graded composite strip, Span direction, Fourier series in complex form Abstract: A graded composite strip with elastic variation in span direction is investigated in the paper. So far, studies about materials variation in span direction are rare. Here, the strip is treated as a plane problem, and the modulus varies exponentially with span direction, and Poisson ratio is constant. By using Fourier decomposition in complex form, the series solution for the strip is obtained and is verified by finite element model.

\section{Introduction}

Many researchers have performed many studies on the mechanics behavior of inhomogeneous beams/strips and related structures [1-3]. Sankar [4] obtained an elasticity solution of a functionally graded beam subjected to a transverse sinusoidal load, in which the elastic modulus exhibits exponential variation through the thickness. Zhong and Yu [5] presented a general solution of a functionally graded beam with arbitrary graded variations of material property by the Airy stress function. Yang [6] investigated the mechanics behavior of a bi-layer functionally graded cantilever beam with concentrated loads, whose elastic modulus of each graded layer can varies with the thickness as an arbitrary function, respectively. An Elastic solution of a FG cantilever beam with different modulus in tension and compression under bending loads is studied by Yang[7]. Wang and Li studied [8] the bending problem of a bi-material beam with a viscoelastic intermediate layer.

There are many researches about beams/strips with modulus varying along thickness, but few works about modulus varying with span direction. In this paper, a graded composite strip, treated as an inhomogeneous plane problem, and with elastic gradation in span direction, is investigated. The strip is subjected to symmetrical transverse loads and its modulus is assumed to vary exponentially in span direction, and its Poisson ratio is hold as a constant. By using Fourier decomposition in complex form in span direction, a closed form solution in series expansion is obtained and verified by finite element results.

\section{Model and basic formulation}

Consider a graded composite strip subjected to symmetrical transverse loads, in a Cartesian coordinate system, in Fig 1.Its modulus vary exponentially along the longitudinal direction and Poisson ratio is hold constant. The thickness and length are $2 h$ and $l$, respectively .

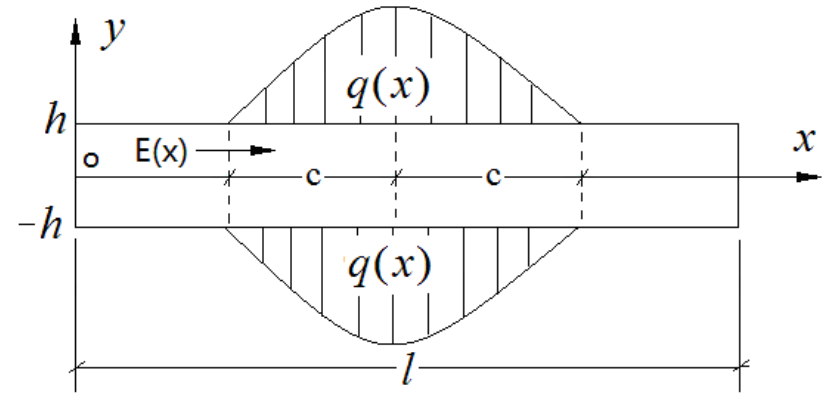

Fig.1 Schematic of graded composite strip. 
The model is treated as plane elasticity problem. Hence, in the absence of body force the equations are given as

$$
\frac{\partial \sigma_{x}}{\partial x}+\frac{\partial \tau_{y x}}{\partial y}=0, \frac{\partial \tau_{y x}}{\partial x}+\frac{\partial \sigma_{y}}{\partial y}=0
$$

where $\sigma_{x}, \sigma_{y}$ and $\tau_{y x}$ represent the stress components.

The relations between strains and displacements are

$$
\varepsilon_{x}=\frac{\partial u}{\partial x}, \varepsilon_{y}=\frac{\partial w}{\partial y}, \gamma_{y x}=\frac{\partial u}{\partial y}+\frac{\partial w}{\partial x}
$$

where $\varepsilon_{x}, \varepsilon_{y}, \gamma_{y x}$ denote the strain components and $u, w$ denote the displacement components. Then, the strain compatibility equation can be derived from Eq. (2.1) as

$$
\frac{\partial^{2} \varepsilon_{x}}{\partial y^{2}}+\frac{\partial^{2} \varepsilon_{y}}{\partial x^{2}}-\frac{\partial^{2} \gamma_{y x}}{\partial y \partial x}=0
$$

The constitutive relations of strip are

$$
\left\{\begin{array}{l}
\varepsilon_{x}=s_{11} \sigma_{x}+s_{12} \sigma_{y} \\
\varepsilon_{y}=s_{21} \sigma_{x}+s_{22} \sigma_{y} \\
\gamma_{y x}=s_{44} \tau_{y x}
\end{array}\right.
$$

where $s_{i j}$ are elastic compliance parameters. Comparing with homogeneous materials, $s_{i j}$ are functions of gradation coordinate. As for two-dimension inhomogeneous problem, $s_{i j}$ can be further described by

$$
s_{i j}=s_{i j}(x, y)
$$

In order to satisfy the equations of equilibrium, the stress components are defined in terms of Airy stress function $\varphi(x, y)$ as

$$
\sigma_{x}=\frac{\partial^{2} \varphi}{\partial y^{2}}, \sigma_{y}=\frac{\partial^{2} \varphi}{\partial x^{2}}, \tau_{y x}=-\frac{\partial^{2} \varphi}{\partial y \partial x}
$$

By substituting Eq.(2.6), Eq.(2.4) into Eq.(2.3) and with concern of Eq.(2.5), we can obtain the governing equation in Airy stress function

$$
\frac{\partial^{2}}{\partial y^{2}}\left(s_{11} \frac{\partial^{2} \varphi}{\partial y^{2}}+s_{12} \frac{\partial^{2} \varphi}{\partial x^{2}}\right)+s_{12} \frac{\partial^{4} \varphi}{\partial y^{2} \partial x^{2}}+\frac{\partial}{\partial y}\left(s_{44} \frac{\partial^{3} \varphi}{\partial x^{2} \partial y}\right)+s_{22} \frac{\partial^{4} \varphi}{\partial x^{4}}=0
$$

Here, the elastic modulus is assumed to vary exponentially along the longitudinal direction. Then, the function of modulus, respected to longitudinal coordinate $x$, can be defined by

$$
E(x)=E_{0} e^{\beta x}
$$

where $E_{0}$ is the basic modulus at $x=0$, and $\beta$ is the inhomogeneous factor in longitudinal direction.

In plane stress problem, the relation between $s_{i j}$ and $E(x)$ can be written as

$$
s_{11}(x)=s_{22}(x)=\frac{1}{E(x)}=\frac{1}{E_{0} e^{\beta x}} ; s_{12}(x)=s_{21}(x)=-\frac{v}{E(x)}=-\frac{v}{E_{0} e^{\beta x}} ; s_{44}(x)=\frac{2(1+v)}{E(x)}=\frac{2(1+v)}{E_{0} e^{\beta x}}
$$

If transfer the problem to plane strain, we can replace $v$ by $v^{*}=\frac{v}{1-v}$ in Eq.(2.8).

Noticing Eqs.(2.7) and (2.8), the governing equation (2.6) of the problem can be given by

$$
\frac{\partial^{4} \varphi}{\partial x^{4}}-2 \beta \frac{\partial^{3} \varphi}{\partial x^{3}}+\beta^{2} \frac{\partial^{2} \varphi}{\partial x^{2}}+2 \frac{\partial^{4} \varphi}{\partial x^{2} \partial y^{2}}-2 \beta \frac{\partial^{3} \varphi}{\partial x \partial y^{2}}-v \beta^{2} \frac{\partial^{2} \varphi}{\partial y^{2}}+\frac{\partial^{4} \varphi}{\partial y^{4}}=0
$$

Eq.(2.9) usually is solved by method of separation of variables. We thus write Airy stress function $\varphi(x, y)$ in the separated-variable form as 
If take $g(x)=e^{i \xi x}$, we have

$$
\varphi(x, y)=g(x) f(y)
$$

$$
\sigma_{x}(x, y)=f^{(2)}(y) e^{i \xi x} ; \sigma_{y}(x, y)=-\xi^{2} f(y) e^{i \xi x} ; \tau_{x y}(x, y)=-i \xi f^{(1)}(y) e^{i \xi x}
$$

It is observed that Eq.(2.11) could satisfy arbitrary traction conditions described by Fourier series expansion in complex form on the boundaries $y= \pm h$. Thus, it may be the solution of the problem.

Now, substituting Eq.(2.10) into Eq.(2.9), we have

$$
\left[f^{(4)}(y)-\left(2 \xi^{2}+2 i \xi \beta+v \beta^{2}\right) f^{(2)}(y)-\xi^{2}(\beta-i \xi)^{2} f(y)\right] e^{(i \xi-\beta) x}=0
$$

Eq.(2.12) is a fourth-order linear differential equations with constant coefficients of $f(y)$, and its characteristic equation can be given by

$$
\omega^{4}-\left(2 \xi^{2}+2 i \xi \beta+v \beta^{2}\right) \omega^{2}-\xi^{2}(\beta-i \xi)^{2}=0
$$

Then, we obtain four characteristic roots about $\omega$

$$
\begin{aligned}
& \omega_{n 1}=\sqrt{\frac{v \beta^{2}+2 \xi_{n}\left(i \beta+\xi_{n}\right)-\beta \sqrt{v^{2} \beta^{2}+4 v \xi_{n}\left(i \beta+\xi_{n}\right)}}{2}} \\
& \omega_{n 2}=-\sqrt{\frac{v \beta^{2}+2 \xi_{n}\left(i \beta+\xi_{n}\right)-\beta \sqrt{v^{2} \beta^{2}+4 v \xi_{n}\left(i \beta+\xi_{n}\right)}}{2}} \\
& \omega_{n 3}=\sqrt{\frac{v \beta^{2}+2 \xi_{n}\left(i \beta+\xi_{n}\right)+\beta \sqrt{v^{2} \beta^{2}+4 v \xi_{n}\left(i \beta+\xi_{n}\right)}}{2}} \\
& \omega_{n 4}=-\sqrt{\frac{v \beta^{2}+2 \xi_{n}\left(i \beta+\xi_{n}\right)+\beta \sqrt{v^{2} \beta^{2}+4 v \xi_{n}\left(i \beta+\xi_{n}\right)}}{2}}
\end{aligned}
$$

It is easy to be proved that when $\beta \neq 0, v \neq 0, \xi \neq 0$, Eq. (2.13) has not multiple roots ( $\beta=0$ denote the case of homogeneous materials.). Therefore, the general solution of $f(y)$ can be written as

$$
f_{n}(y)=C_{n 1} e^{\omega_{n 1} y}+C_{n 2} e^{\omega_{n 2} y}+C_{n 3} e^{\omega_{n 3} y}+C_{n 4} e^{\omega_{n 4} y}
$$

where $C_{n j}(j=1,2,3,4)$ are complex constants to be determined.

Therefore, the stress function $\varphi(x, y)$ can be determined by Eqs.(2.13) and (2.19)

$$
\varphi(x, y)=e^{i \xi x}\left[C_{1} e^{\omega_{1} y}+C_{2} e^{\omega_{2} y}+C_{3} e^{\omega_{3} y}+C_{4} e^{\omega_{4} y}\right]
$$

\section{The series solutions of a graded composite strip}

Consider a graded composite strip subjected to transverse loads in Fig.1 and its boundary conditions is defined by

$$
\sigma_{y}(x, h)=\sigma_{y}(x,-h)=q(x), \tau_{x y}(x, h)=\tau_{x y}(x,-h)=0
$$

In coordinate system given in Fig.1, the traction loads can be seen as odd functions, therefore, the loads Eq.(3.1) can be expanded by Fourier series in complex form as

with

$$
q(x)=\sum_{n=-\infty}^{\infty} c_{n} e^{i \frac{n \pi x}{l}}
$$

$$
c_{n}=\frac{-i}{l} \int_{0}^{l} q(x) \sin \left(\frac{n \pi x}{l}\right) d x, \quad n= \pm 1, \pm 2, \pm 3, \cdots
$$

In order to satisfy the boundary conditions in the form of series, the stress function can be given in the form of superposition of terms as

$$
\varphi(x, y)=\sum_{n=-\infty}^{\infty}\left(C_{n 1} e^{\omega_{n 1} y}+C_{n 2} e^{\omega_{n 2} y}+C_{n 3} e^{\omega_{n 3} y}+C_{n 4} e^{\omega_{n 4} y}\right) e^{i \xi_{n} x}
$$


where $\xi_{n}=\frac{n \pi}{l}$. Then, the stress components can be derived as

$$
\begin{gathered}
\sigma_{x}(x, y)=\sum_{n=-\infty}^{\infty}\left(C_{n 1} \omega_{n 1}{ }^{2} e^{\omega_{n 1} y}+C_{n 2} \omega_{n 2}{ }^{2} e^{\omega_{n 2} y}+C_{n 3} \omega_{n 3}{ }^{2} e^{\omega_{n 3} y}+C_{n 4} \omega_{n 4}{ }^{2} e^{\omega_{n 4} y}\right) e^{i \xi_{n} x} \\
\sigma_{y}(x, y)=-\sum_{n=-\infty}^{\infty} \xi_{n}{ }^{2}\left(C_{n 1} e^{\omega_{n 1} y}+C_{n 2} e^{\omega_{n 2} y}+C_{n 3} e^{\omega_{n 3} y}+C_{n 4} e^{\omega_{n 4} y}\right) e^{i \xi_{n} x} \\
\tau_{x y}(x, y)=-\sum_{n=-\infty}^{\infty} i \xi_{n}\left(C_{n 1} \omega_{n 1} e^{\omega_{n 1} y}+C_{n 2} \omega_{n 2} e^{\omega_{n 2} y}+C_{n 3} \omega_{n 3} e^{\omega_{n 3} y}+C_{n 4} \omega_{n 4} e^{\omega_{n 4} y}\right) e^{i \xi_{n} x}
\end{gathered}
$$

For convenience, the stress function and stress components can be expressed in the form of trigonometric function. It is observed from Eq. $(2.14)$ that $\omega_{n j}(\mathrm{j}=1,2,3,4)$ are complex, and $\omega_{n 1}$ and $\omega_{n 2}$ are a pair of opposite number, and $\omega_{n 1}$ and $\omega_{n 2}$ are another pair of opposite number. So we have

$$
\left\{\begin{array}{l}
\omega_{n 1}=\mu_{n}+\theta_{n} i \\
\omega_{n 2}=-\mu_{n}-\theta_{n} i \\
\omega_{n 3}=\eta_{n}+\theta_{n} i \\
\omega_{n 4}=-\eta_{n}-\theta_{n} i
\end{array}\right.
$$

By using Euler formulation $\left\{\begin{array}{l}e^{i x}=\cos (x)+i \sin (x) \\ e^{-i x}=\cos (x)-i \sin (x)\end{array}\right.$, the stress function in trigonometric form can be obtained

$$
\begin{aligned}
\varphi(x, y)= & \sum_{n=-\infty}^{\infty}\left[C_{n 1} e^{\mu_{n} y}\left(\cos \left(\theta_{n} y\right)+i \sin \left(\theta_{n} y\right)\right)+C_{n 2} e^{-\mu_{n} y}\left(\cos \left(\theta_{n} y\right)-i \sin \left(\theta_{n} y\right)\right)\right. \\
& \left.+C_{n 3} e^{\eta_{n} y}\left(\cos \left(\theta_{n} y\right)+i \sin \left(\theta_{n} y\right)\right)+C_{n 4} e^{-\eta_{n} y}\left(\cos \left(\theta_{n} y\right)-i \sin \left(\theta_{n} y\right)\right)\right] e^{i \xi_{n} x}
\end{aligned}
$$

The consequent stress components are

$$
\begin{gathered}
\sigma_{x}(x, y)=\sum_{n=-\infty}^{\infty} e^{i \xi_{n} x}\left[C_{n 1} e^{\mu_{n} y}\left(\mu_{n}+\theta_{n} i\right)^{2}\left(\cos \left(\theta_{n} y\right)+i \sin \left(\theta_{n} y\right)\right)+\right. \\
C_{n 2} e^{-\mu_{n} y}\left(\mu_{n}+\theta_{n} i\right)^{2}\left(\cos \left(\theta_{n} y\right)-i \sin \left(\theta_{n} y\right)\right)+ \\
C_{n 3} e^{\eta_{n} y}\left(\eta_{n}+\theta_{n} i\right)^{2}\left(\cos \left(\theta_{n} y\right)+i \sin \left(\theta_{n} y\right)\right)+ \\
\left.C_{n 4} e^{-\eta_{n} y}\left(\eta_{n}+\theta_{n} i\right)^{2}\left(\cos \left(\theta_{n} y\right)-i \sin \left(\theta_{n} y\right)\right)\right] \\
\sigma_{y}(x, y)=-\sum_{n=-\infty}^{\infty} \xi_{n}{ }^{2} e^{i \xi_{n} x}\left[C_{n 1} e^{\mu y}\left(\cos \left(\theta_{n} y\right)+i \sin \left(\theta_{n} y\right)\right)+\right. \\
C_{n 2} e^{-\mu_{n} y}\left(\cos \left(\theta_{n} y\right)-i \sin \left(\theta_{n} y\right)\right)+ \\
C_{n 3} e^{\eta_{n} y}\left(\cos \left(\theta_{n} y\right)+i \sin \left(\theta_{n} y\right)\right)+ \\
\left.C_{n 4} e^{-\eta_{n} y}\left(\cos \left(\theta_{n} y\right)-i \sin \left(\theta_{n} y\right)\right)\right] \\
\left.C_{x y}^{\infty} y\right)=-\sum_{n=-\infty}^{\infty} i \xi_{n} e^{i \xi_{n} x}\left[C_{n 1} e^{\mu y}\left(\mu_{n}+\theta_{n} i\right)\left(\cos \left(\theta_{n} y\right)+i \sin \left(\theta_{n} y\right)\right)-\right. \\
C_{n 2} e^{-\mu_{n} y}\left(\mu_{n}+\theta i\right)\left(\cos \left(\theta_{n} y\right)-i \sin \left(\theta_{n} y\right)\right)+ \\
C_{n 3} e^{\eta_{n} y}\left(\eta_{n}+\theta_{n} i\right)\left(\cos \left(\theta_{n} y\right)+i \sin \left(\theta_{n} y\right)\right)- \\
\left.C_{n 4} e^{-\eta y}\left(\eta_{n}+\theta_{n} i\right)\left(\cos \left(\theta_{n} y\right)-i \sin \left(\theta_{n} y\right)\right)\right]
\end{gathered}
$$

According to the traction conditions (3.1) and noticing Eq.(3.7), we have a set of equations 


$$
\begin{gathered}
C_{n 1} e^{\mu_{n} h}\left(\cos \left(\theta_{+n} h\right)+i \sin \left(\theta_{n} h\right)\right)+C_{n 2} e^{-\mu_{n} h}\left(\cos \left(\theta_{n} h\right)-i \sin \left(\theta_{n} h\right)\right) \\
+C_{n 3} e^{\eta_{n} h}\left(\cos \left(\theta_{n} h\right)+i \sin \left(\theta_{n} h\right)\right)+C_{n 4} e^{-\eta_{n} h}\left(\cos \left(\theta_{+n} h\right)-i \sin \left(\theta_{n} h\right)\right)=-c_{n} \\
C_{n 1} e^{-\mu_{n} h}\left(\cos \left(\theta_{n} h\right)-i \sin \left(\theta_{n} h\right)\right)+C_{n 2} e^{\mu_{n} h}\left(\cos \left(\theta_{n} h\right)+i \sin \left(\theta_{n} h\right)\right) \\
+C_{n 3} e^{-\eta_{n} h}\left(\cos \left(\theta_{n} h\right)-i \sin \left(\theta_{n} h\right)\right)+C_{n 4} e^{\eta_{n} h}\left(\cos \left(\theta_{n} h\right)+i \sin \left(\theta_{n} h\right)\right)=-c_{n} \\
C_{n 1} e^{\mu_{n} h}\left(\mu_{n}+\theta_{n} i\right)\left(\cos \left(\theta_{n} h\right)+i \sin \left(\theta_{n} h\right)\right)-C_{n 2} e^{-\mu_{n} h}\left(\mu_{n}+\theta_{n} i\right)\left(\cos \left(\theta_{n} h\right)-i \sin \left(\theta_{n} h\right)\right)+ \\
C_{n 3} e^{\eta_{n} h}\left(\eta_{n}+\theta_{n} i\right)\left(\cos \left(\theta_{n} h\right)+i \sin \left(\theta_{n} h\right)\right)-C_{n 4} e^{-\eta_{n} h}\left(\eta_{n}+\theta_{n} i\right)\left(\cos \left(\theta_{n} h\right)-i \sin \left(\theta_{n} h\right)\right)=0 \\
C_{n 1} e^{-\mu_{n} h}\left(\mu_{n}+\theta_{n} i\right)\left(\cos \left(\theta_{n} h\right)-i \sin \left(\theta_{n} h\right)\right)-C_{n 2} e^{\mu_{n} h}\left(\mu_{n}+\theta_{n} i\right)\left(\cos \left(\theta_{n} h\right)+i \sin \left(\theta_{n} h\right)\right) \\
+C_{n 3} e^{-\eta_{n} h}\left(\eta_{n}+\theta_{n} i\right)\left(\cos \left(\theta_{n} h\right)-i \sin \left(\theta_{n} h\right)\right)-C_{n 4} e^{\eta_{n} h}\left(\eta_{n}+\theta_{n} i\right)\left(\cos \left(\theta_{n} h\right)+i \sin \left(\theta_{n} h\right)\right)=0
\end{gathered}
$$

We can solve 4 equations for 4 unknowns from Eqs.(3.8), then we obtain the elastic solution of a graded composite strip with modulus varying along longitudinal direction.

\section{Example and discussion}

Solve the strip in Fig 1 . Take $l=20, h=1, c=1, v=0.3$, and assume $E_{0}=100 M P a$. The boundary conditions of the problem are addressed by

$$
q(x)=\left\{\begin{array}{rll}
0 & , & 0 \leq x<l / 2-1 \\
\sin \left(\frac{\pi x}{2}\right) & , & l / 2-1 \leq x \leq l / 2+1 \\
0 & , & l / 2+1<x \leq l
\end{array}\right.
$$

which depicts a discontinuous pressure load.

On the upper and lower edges of the strip, the distributions of normal stress $\sigma_{y}$ satisfy Eq.(4.2) which is symmetric with the cross section $x=10$ that is the position with the maximum pressure, as shown in Fig 2,3

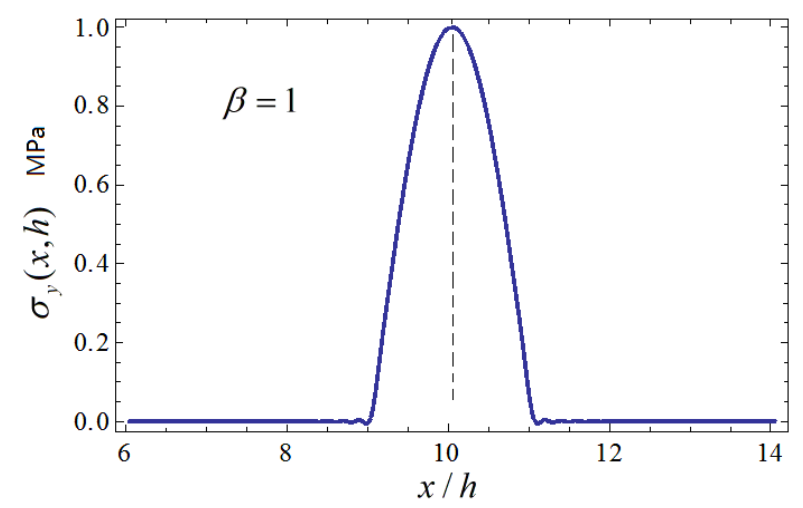

Fig.2 The distribution of $\sigma_{y}$ on the upper edges of strip

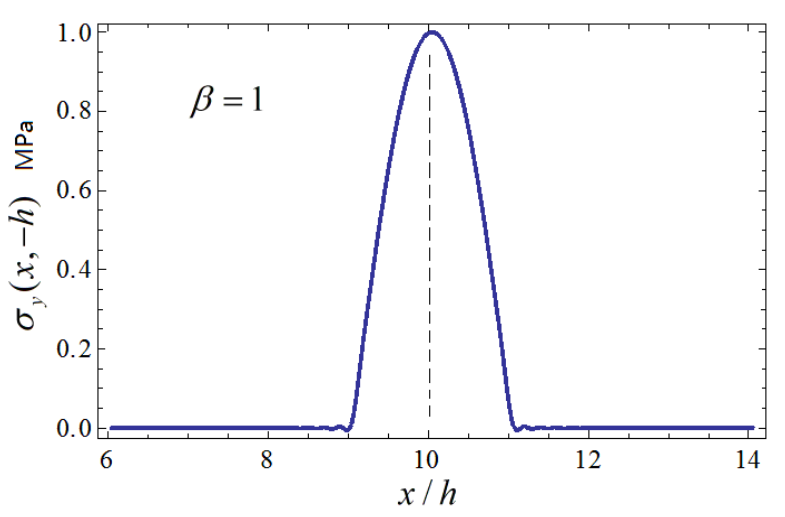

Fig. 3 The distribution of $\sigma_{y}$ on the lower edges of strip

Fig.4 and Fig.5 show the distributions of stress $\sigma_{y}$ in $\beta=1,2,3$ and $\beta=-1,-2,-3$, respectively. It is clear from Fig.12 that the biggest stress $\sigma_{y}$ don't occur on the cross section $x=10$ with the biggest pressure, and its position moves toward the direction of bigger modulus, with $\beta$ changing. The symmetry of the normal stress distribution is destroyed by inhomogeneity of materials, which is different from the case of homogeneity. It is also can be seen that Fig.4 and Fig. 5 are symmetric with each other. When $\beta>0$, the curves in the right part of Fig. 4 experience a process that decay from positive value to negative value firstly, then they vanish, and vice versa in 
the case of $\beta<0$.

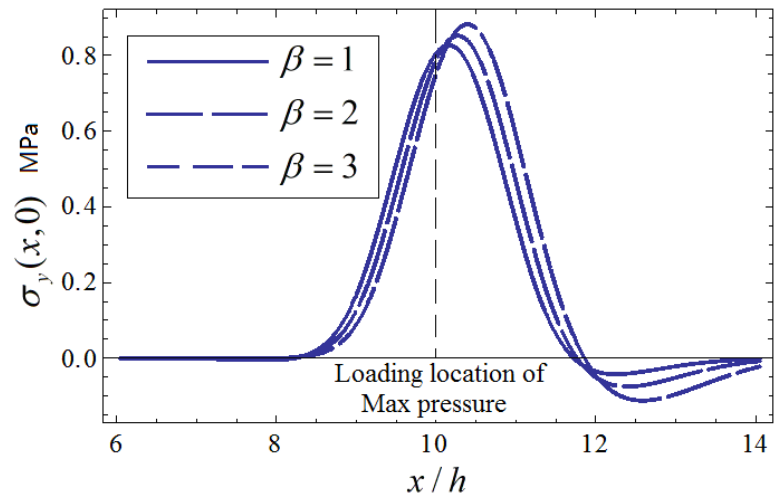

Fig.4 The influence of $\beta$ on $\sigma_{y}$

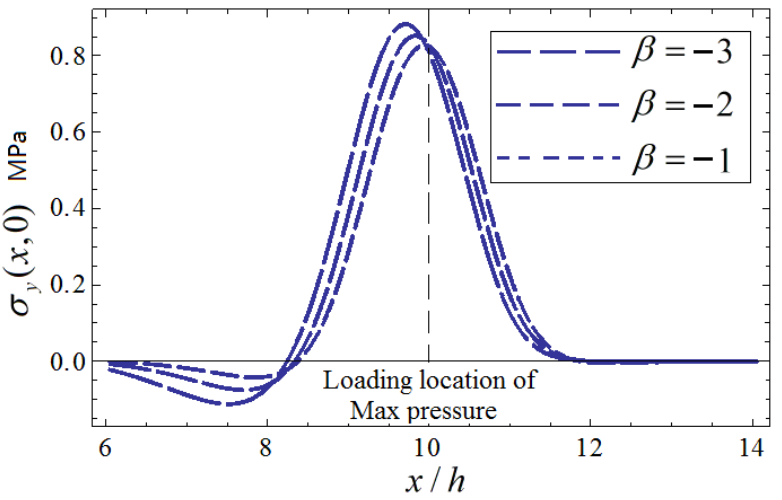

Fig. 5 The influence of $\beta$ on $\sigma_{y}$

The contour plots of stress distribution are calculated by FEM and series methods, respectively, as shown in Fig 13

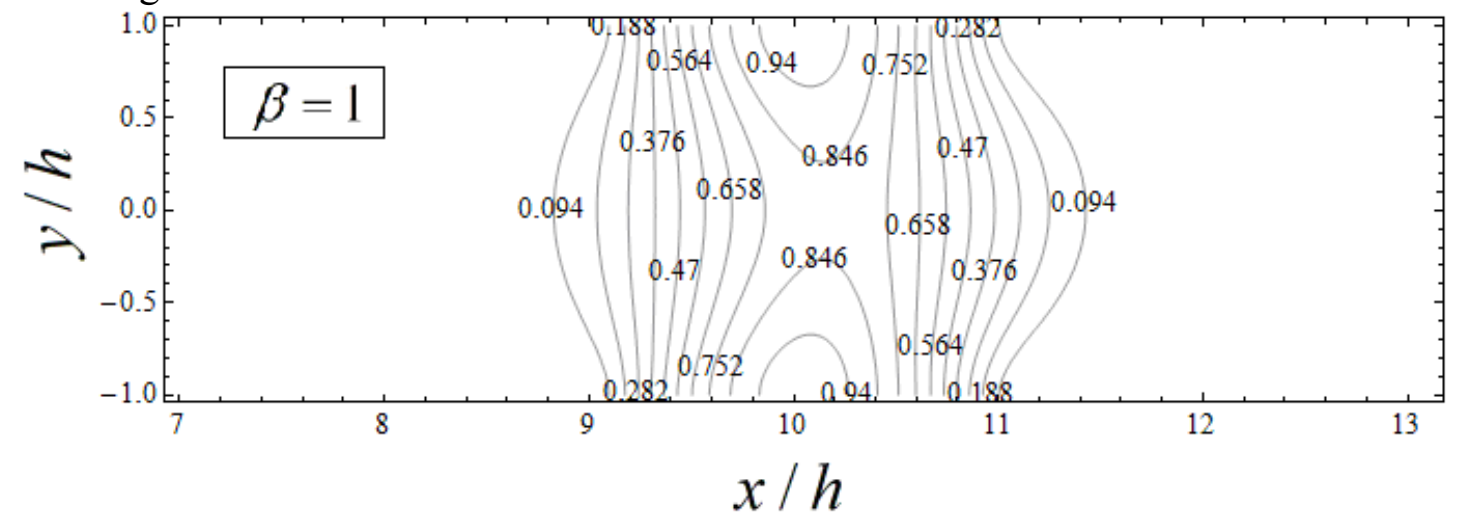

Fig. 6 Contour Plot of $\sigma_{y}$ by theory method

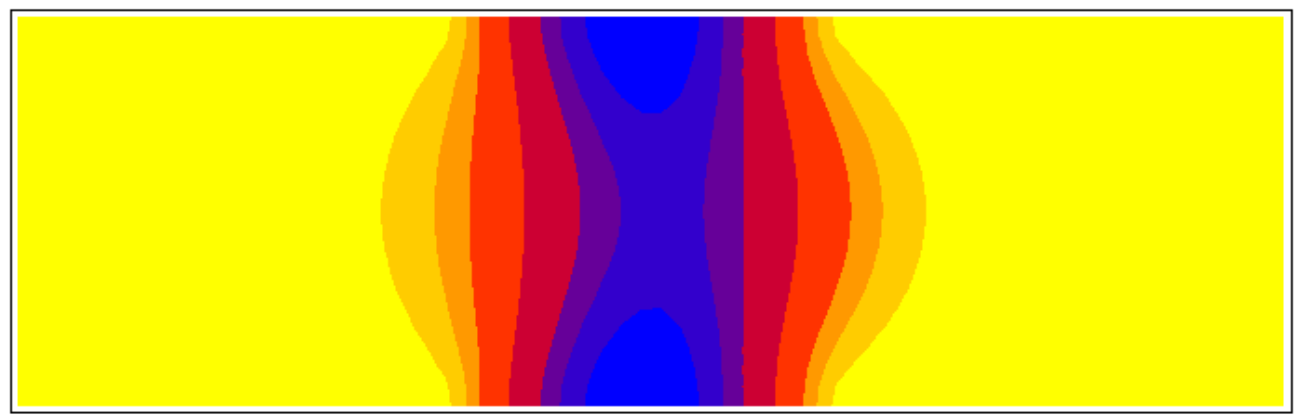

Fig.7 Contour Plot of $\sigma_{y}$ by FEM

From Figs.6 and 7, it is clear that the stress field is asymmetric with the line of the maximum pressure loading and tends to move toward the direction with bigger modulus. The errors between FEM results and theory results seem very small, which can be verified by Fig. 8 


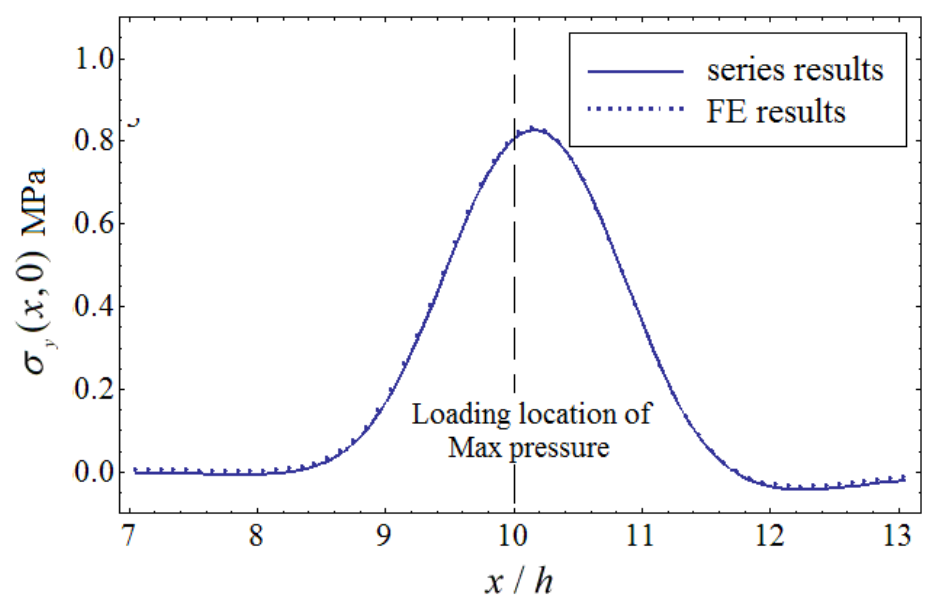

Fig.8 The FEM and series results of $\sigma_{y}$ on the plane $y=0$

\section{Conclusions}

In this paper, a graded composite strip with elastic gradation in span direction is investigated by Fourier decomposition in complex form. Firstly, a general solution of a fourth-order governing equation by Airy function is obtained. Immediately, a series solution for the problem of the strip subjected to transverse loads is derived. From a example proposed here, it can be found that the inhomogeneity of materials, along span direction, has a significant influence on the mechanics behavior of a strip. Especially, when the strip subjected to a pair of symmetrical normal tractions on the upper and lower boundaries, the biggest stress within the strip don't occur on the location of the maximum traction loading, while moves toward the direction with bigger modulus. Comparing the analytical solutions in series form with FEM results, a good agreement has achieved.

\section{Acknowledgements}

This work was supported by National international scientific and technological cooperation projects of China (No. 2013DFG52420) and the project of cooperation of China and EU (SAMC14-JS-13-012).

\section{References}

[1]. S Suresh., A Mortensen, Fundamentals of functionally graded materials., IOM Communications Limited, London, 1998.

[2]. D. Zenkert, The Handbook of Sandwich Construction., Materials Advisory Services Ltd, London, 1997.

[3]. S. Abrate, Impact on Composite Structures., Cambridge University Press, Cambridge, 1998.

[4]. B.V. Sankar, Compos. Sci. Tech. 61 (2001) 689-696.

[5]. Z. Zhong, T Yu, Compos. Sci. Tech. 67 ( 2007) 481-8.

[6]. Q. Yang, B.L. Zheng, K Zhang and J.X. Zhu, Arch. Appl. Mech. 83(2013) 455 466.

[7]. Q. Yang, B.L. Zheng, K. Zhang and J.X. Zhu, Appl. Math. Model. 38(2014) 1403-16.

[8]. J. Li, B.L. Zheng, Q Yang and X.J. Hu, Compos. Struct.107 (2014) 30-35. 